\title{
Inverse Limit Spaces with Various Shadowing Property
}

\author{
Ali Barzanouni \\ Department of Mathematics and Computer Sciences, Hakim Sabzevari University, P.O. Box 397, Sabzevar, Iran
}

Correspondence should be addressed to Ali Barzanouni; alibarzanouni@yahoo.com

Received 19 May 2014; Accepted 13 August 2014; Published 14 September 2014

Academic Editor: Francisco B. Gallego

Copyright (C) 2014 Ali Barzanouni. This is an open access article distributed under the Creative Commons Attribution License, which permits unrestricted use, distribution, and reproduction in any medium, provided the original work is properly cited.

We discuss the relationship between ergodic shadowing property and inverse shadowing property of $f$ and that of the shift map $\sigma_{f}$ on the inverse limit space.

\section{Introduction}

The notion of pseudoorbits and pseudoorbit shadowing property often appears in several branches of modern theory of dynamical systems (see [1]) and usually plays an important role in the investigation of stability theory and ergodic theory. The authors [2] introduced the notion of ergodic shadowing for a continuous onto map which is equivalent to the map being topologically mixing and has the ordinary shadowing property and deduces the chaotic behavior of a map with ergodic shadowing property. In [3], the authors proved that if any $\delta$-ergodic pseudoorbit of a system is shadowed by a point along a set of positive lower density, then this system is chain mixing and if it is minimal, then it is topologically weakly mixing and so is Li-Yorke chaos. Recently, in [4], the notion of weakly ergodic shadowing property was introduced; the author showed that weakly ergodic shadowing property is equivalent to ergodic shadowing property. If $f$ has ergodic shadowing property (ESP), then it has shadowing property (SP), but as we will see the converse is not true; that is, there are systems with shadowing property which do not have ergodic shadowing property (see Example 3). Therefor ergodic shadowing property is not equivalent to shadowing property. Inverse limits of maps have been studied for decades, as a means of studying both continua and dynamical systems. In 2004 and 2006, Mahavier [5] and Ingram and Mahavier [6] introduced the concept of inverse limits with upper semicontinuous set-valued functions. Inverse limit is a useful tool to study the dynamical properties of smooth systems; some dynamical properties of $f$ can be interpreted by the topological structures of the inverse limit dynamical system; for example, Chen and $\mathrm{Li}$ [7] proved that $(X, f)$ has the shadowing property if and only if $\left(\lim _{\leftarrow}(X, f), \sigma_{f}\right)$ has so. Li [8] proved that some dynamical properties hold simultaneously for both $f$ and $\sigma_{f}$. In this paper, we discuss the relationship between ergodic shadowing property and inverse shadowing property for a surjective continuous map on a compact metric space and shift map on the inverse limit space.

\section{Definitions}

Let $X$ be a compact metric space with metric $d$ and let $f$ : $X \rightarrow X$ be a continuous map. For every $n \in \mathbb{N}$, $f^{n}$ is defined inductively by $f^{n}=f o f^{n-1}$, where $f^{0}$ is the identity map on $X$. For $\delta>0$, a sequence $\left\{x_{i}\right\}_{a \leq i \leq b}$ is called a $\delta$ pseudoorbit of $f$ if $d\left(f\left(x_{i}\right), x_{i+1}\right)<\delta$ for every $a \leq i \leq b$. If, for any $x, y \in X$ and every $\epsilon>0$, there is a finite $\epsilon$ pseudoorbit $\left\{x_{0}, x_{1}, \ldots, x_{n}\right\}$ of $X$ such that $x_{0}=x, x_{n}=y$, then $\left\{x_{0}, x_{1}, \ldots, x_{n}\right\}$ is called an $\epsilon$-chain from $x$ to $y$, and $n+1$ is called the length of the $\epsilon$-chain. If, for any $\epsilon>0$ and every $x, y \in X$, there is an $\epsilon$-chain from $x$ to $y$, then $f$ is called chain transitive. Moreover if there is a positive integer $N$, such that when $n>N$, there is an $\epsilon$-chain with length $n$ from $x$ to $y$, then $f$ is called chain mixing. For any nonempty open sets $U$ and $V$ if there is $n>0$ such that $f^{n}(U) \cap V \neq \emptyset$, then $f$ is called topologically transitive and if there is $N>0$, such that, for any $n>N, f^{n}(U) \cap V \neq \emptyset$, then $f$ is called topologically mixing. Obviously, topologically transitive (mixing) map is chain transitive (mixing). Let $x, y \in X$ be given. We write $x \stackrel{f}{\rightarrow} y$ if for every $\delta>0$ there is a $\delta$-pseudoorbit $\left\{x_{i}\right\}_{i=0}^{l}$ 
of some length $l+1$ of $f$ such that $x=x_{0}, \ldots, x_{n}=y$. We write $x \stackrel{f}{\sim} y$ if $x \stackrel{f}{\rightarrow} y$ and $y \stackrel{f}{\rightarrow} x$. A sequence $\left\{x_{i}\right\}_{i \in \mathbb{Z}}$ (resp., $\left\{x_{i}\right\}_{i \in \mathbb{N}}$ ) in $X$ is said to be $\epsilon$-traced by some point $z$ in $X$ if $d\left(f^{i}(z), x_{i}\right)<\epsilon$ for each integer $i \in \mathbb{Z}$ (resp., for every $i \in \mathbb{N}$ ). A map $f$ is said to have the shadowing property, SP (resp., $\mathrm{SP}^{+}$), if for any $\epsilon>0$ there is $\delta>0$ such that every $\delta$ pseudoorbit of $f$ can be $\epsilon$-shadowed by some point in $X$. Let $X^{\mathbb{Z}}$ be the compact metric space of all two-sided sequences $\xi=\left\{x_{k}: k \in \mathbb{Z}\right\}$ in $X$, endowed with the product topology. For a constant $\delta>0$ and $f: X \rightarrow X$, let $\Phi_{f}(\delta)$ denote the set of all $\delta$-pseudoorbits of $f$. A mapping $\varphi: X \rightarrow \Phi_{f}(\delta) \subseteq X^{\mathbb{Z}}$ satisfying $\varphi_{o}(x)=x, x \in X$, is said to be a $\delta$-method for $f$. For convenience, write $\varphi(x)$ for $\left\{\varphi_{k}(x)\right\}_{k \in \mathbb{Z}}$.

$f: X \rightarrow X$ is said to be inverse shadowing, denoted by ISP (resp., positive inverse shadowing property, $\mathrm{ISP}^{+}$) if for any $\epsilon>0$ there exists $\delta>0$ such that for any $x \in X$ and any $\delta$ method $\varphi: X \rightarrow X^{\mathbb{Z}}$ (resp., $\varphi: X \rightarrow X^{\mathbb{N}}$ ) there is $y \in X$ such that $d\left(f^{k}(x), \varphi_{k}(y)\right)<\epsilon$, for all $k \in \mathbb{Z}$ (resp., for all $k \in \mathbb{N})$.

Given a sequence $\xi=\left\{x_{i}\right\}_{i \in \mathbb{Z}}$ (resp., $\xi=\left\{x_{i}\right\}_{i \in \mathbb{N}}$ ), put $N P O(\xi, f, \delta):=\left\{i \in \mathbb{Z}: d\left(f\left(x_{i}\right), x_{i+1}\right) \geq \delta\right\}$ (resp., $\left.\operatorname{NPO}^{+}(\xi, f, \delta):=\left\{i \in \mathbb{N}: d\left(f\left(x_{i}\right), x_{i+1}\right) \geq \delta\right\}\right)$ and $N \mathrm{NO}_{n}(\xi, f, \delta):=\operatorname{NPO}(\xi, f, \delta) \bigcap\{-n, \ldots,-2,-1,0,1,2, \ldots$, $n\}$ (resp., $\left.N P O_{n}^{+}(\xi, f, \delta):=N P O^{+}(\xi, f, \delta) \bigcap\{1,2,3, \ldots, n\}\right)$.

For a sequence $\xi=\left\{x_{i}\right\}_{i \in \mathbb{Z}}$ (resp., $\xi=\left\{x_{i}\right\}_{i \in \mathbb{N}}$ ) and a point $x$ of $X$, put $N S(\xi, x, f, \delta):=\left\{i \in \mathbb{Z}: d\left(f^{i}(x), x_{i}\right) \geq \delta\right\}$ (resp., $N S^{+}(\xi, x, f, \delta):=\left\{i \in \mathbb{N}: d\left(f^{i}(x), x_{i}\right) \geq \delta\right\}$ ) and $N S_{n}(\xi, x, f, \delta):=N S(\xi, x, \delta) \bigcap\{-n, \ldots,-2,-1,0,1,2, \ldots, n\}$ (resp., $\left.N S_{n}^{+}(\xi, x, f, \delta):=N S^{+}(\xi, x, f, \delta) \bigcap\{1,2, \ldots, n\}\right) . \xi=$ $\left\{x_{i}\right\}_{i \in \mathbb{Z}}$ (resp., $\xi=\left\{x_{i}\right\}_{i \in \mathbb{N}}$ ) is called a $\delta$-ergodic pseudoorbit for $f$ if $\lim _{|n| \rightarrow \infty}\left(\operatorname{card}\left(N P O_{n}(\xi, f, \delta)\right) / n\right)=0$ (resp., $\lim _{n \rightarrow \infty}$ $\left.\left(\operatorname{card}\left(N P O_{n}^{+}(\xi, f, \delta)\right) / n\right)=0\right)$. A $\delta$-ergodic pseudoorbit is said to be $\epsilon$-ergodic shadowed by a point $x$ in $X$, denoted by $\operatorname{ESP}\left(\right.$ resp., $\left.\operatorname{ESP}^{+}\right)$if $\lim _{|n| \rightarrow \infty}\left(\operatorname{card}\left(N S_{n}(\xi, x, f, \delta)\right) / n\right)=$ 0 (resp., $\left.\lim _{n \rightarrow \infty}\left(\operatorname{card}\left(N S_{n}^{+}(\xi, x, f, \delta)\right) / n\right)=0\right)$. For an $f$ invariant set $\Lambda$, we say that $\Lambda$ has ergodic shadowing property (or $\Lambda$ is ergodic shadowable for $f$ ) if for every $\epsilon>0$ there exists $\delta>0$ such that every $\delta$-ergodic pseudoorbit in $\Lambda$ could be $\epsilon$ ergodic shadowed by a point $x$ in $X$. The following lemma is proved in [2].

Proposition 1. Suppose $f: X \rightarrow X$ be a continuous mapping and surjective on compact metric space $X$. For the dynamical system $(X, f)$, the following properties are equivalent: (a) ergodic shadowable and (b) shadowable and topological mixing.

We do not recall here the definition of topological entropy, since it is well known (e.g., see [9]). For our purpose it is only important to remember that topological entropy is a number $h_{\text {top }}(f) \in[0,+\infty]$ and for every transitive map $f:[0,1] \rightarrow[0,1]$ it is at least $h_{\text {top }}(f) \geq(\log 2 / 2)$ (see Corollary 3.6 in [10]). It is known that every one-dimensional compact connected manifold is homeomorphic with $[0,1]$ or $S^{1}$. The following proposition shows that there exists no homeomorphism of $I=[0,1]$ or $S^{1}$ with ESP. thus in this paper the space $X$ cannot be a one-dimensional connected compact space.

Proposition 2. (1) There exists no homeomorphism $f$ : $[0,1] \rightarrow[0,1]$ with topological mixing.

(2) Every homeomorphism $f: S^{1} \rightarrow S^{1}$ has entropy 0 .

Proof. We prove item (1) but (2) follows from Theorem 7.14 in [11]. Let $f:[0,1] \rightarrow[0,1]$ be a continuous surjective map which is topologically mixing. Since $f$ is onto, there exists $x \in[0,1]$ such that $f(x)=x$. Let $x \in(0,1)$. Choose open intervals $I, J \subset[0,1]$ such that, for every $y \in I$ and $z \in J$, $y<x<z$. For open intervals $I, J$ there exists $N \in \mathbb{N}$ such that if $n \geq N$, then $f^{n}(I) \bigcap J \neq \emptyset$ and $f^{n}(I) \bigcap I \neq \emptyset$. Thus there exist $y \in I$ and $z \in J$ such that $z, y \in f^{n}(I)$ and $y<x<z$. Hence using intermediate value theorem there exists $p \in I$ such that $f^{n}(p)=x$; therefore $f:[0,1] \rightarrow[0,1]$ is not one to one. If $x=0$ or 1 , since $f$ is topologically mixing, it is easy to see that there exists another fixed point in $(0,1)$; thus, in any case, $f:[0,1] \rightarrow[0,1]$ is not one to one.

We know that if $f$ has ergodic shadowing property, then it has one chain component and SP. But the converse is not true. To see this we use an example of [12] to introduce a map which is shadowable but does not have ergodic shadowing property.

Example 3. Let $X=\left\{x=\left\{x_{j}\right\}_{j \in \mathbb{Z}}: x_{j} \in\{0,1\}\right\}$, with the following metric:

$$
d(x, y)= \begin{cases}2, & x_{0} \neq y_{0} \\ \frac{1}{\min \left\{|k|: x_{k} \neq y_{k}\right\}}, & x_{0}=y_{0} .\end{cases}
$$

Let $\pi:\{-n,-n+1, \ldots, n\} \rightarrow\{-n,-n+1, \ldots, n\}$ be a permutation of the set $\{-n,-n+1, \ldots, n\}$ for some $n \in N$. Let $f_{n}(x)=x_{\pi(i)}$ if $-n \leq i \leq n$, and $x_{i}$ otherwise. $f_{n}$ is a homeomorphism and every point of $X$ is a periodic point for $f_{n}$. Let $\delta>0$ be arbitrary and consider $k_{0}:=\min \{k \in \mathbb{N}$ : $(1 / k)<\delta\}$. Then $d(x, y)<\delta$ if and only if $x_{i}=y_{i}$, where $-k_{0}<i<k_{0}$. Hence $x \stackrel{f_{n}}{\sim} y$ if and only if $x=y$. So the chain components of $f_{n}$ are the singletons, and hence $f_{n}$ does not have ergodic shadowing property. But one can easily see that $f_{n}$ has the shadowing property.

Proposition 4. If $f:[0,1] \rightarrow[0,1]$ is continuous and has only one fixed point at endpoint interval, then $f$ has $\mathrm{ISP}^{+}$.

To prove the proposition, we need the following lemma; see $([7])$.

Lemma 5. Let $X$ be a compact metric space and let $f: X \rightarrow$ $X$ be a continuous map. Given $\epsilon>0$ and $M>0$, there exists $\delta>0$ such that every $\delta$-pseudoorbit $\left\{x_{n}\right\}_{n \in \mathbb{N}}$ satisfies $d\left(f^{M}\left(x_{k}\right), x_{k+M}\right)<\epsilon$ for all $k \geq 0$.

Proof of Proposition 4. Let $f(0)=0$ and for every $x \in(0,1]$, $f(x)<x$. For every $\epsilon \in(0,1]$, let $U_{\epsilon}=[0, \epsilon)$ and $\gamma_{\epsilon}=\min \{\mid x-$ $f(x) \mid\}$ for $x \in[\epsilon / 2,1]$; thus $\gamma_{\epsilon}>0$ and $f(x) \leq x-\gamma_{\epsilon}$ for all $x \epsilon$ $[\epsilon / 2,1]$. If $f(x) \in[\epsilon / 2,1]$, then $f^{2}(x) \leq f(x)-\gamma_{\epsilon} \leq x-2 \gamma_{\epsilon}$. 
Let $N>\left((2-\epsilon) / 2 \gamma_{\epsilon}\right)$; we have $f^{n}(x) \in U_{\epsilon / 2}$ for every $x \in[0,1]$ and $n \geq N$. By Lemma 5 for $(\epsilon / 2)>0$ and $N>0$ there exists $\tau>0$ such that if $\left\{z_{n}\right\}_{n \in \mathbb{Z}}$ is a $\tau$-pseudoorbit of $f$, then $\left|z_{n}-f^{N}\left(z_{0}\right)\right| \leq(\epsilon / 2)$; thus $z_{N} \in U_{\epsilon}$. By the uniform continuity of $f$, there exists $\sigma>0$ such that every $\sigma$-pseudoorbit $\left\{z_{n}\right\}_{n \in \mathbb{Z}}$ satisfies $\left|f^{j}\left(z_{0}\right)-z_{j}\right| \leq(\epsilon / 2)$ for every $0 \leq j \leq N$ and there exists $\eta>0$ such that if $|x-y|<\eta$, then $\left|f^{j}(x)-f^{j}(y)\right|<(\epsilon / 2)$ for $0 \leq j \leq N$. Choose $\delta \in\left\{0, \min \left\{\tau, \sigma, \gamma_{\epsilon}, \eta,\left(\epsilon \gamma_{\epsilon} \epsilon\right) /(2-\epsilon)\right\}\right\}$; let $\varphi:[0,1] \rightarrow \phi_{f}(\delta)$ be a $\delta$-method of $f$. If $\varphi(x)=\left\{y_{n}\right\}_{n \in \mathbb{N}}$, then for $n \geq N$, we have $y_{n} \in U_{\epsilon}$. Since $f^{n}(x) \in U_{\epsilon / 2}$ for $n \geq N,\left|f^{n}(x)-y_{n}\right|<\epsilon$ such that $y_{n} \in U_{\epsilon}$. Since $f^{n}(x) \in U_{\epsilon / 2}$ for $n \geq N,\left|f^{n}(x)-y_{n}\right|<\epsilon$ such that $n \geq N$. But for $0 \leq j \leq N$ and $\sigma$-pseudoorbit $\left\{y_{n}\right\},\left|f^{j}\left(y_{0}\right)-y_{j}\right|<(\epsilon / 2)$; this means that, for $n \in \mathbb{N},\left|f^{n}(x)-y_{n}\right|<\epsilon$.

The following proposition is proved in [7].

Proposition 6. If $f:[0,1] \rightarrow[0,1]$ is continuous and has fixed points only at the end of interval, then $f$ has shadowing property.

It is known from [13] that the shift homeomorphism has shadowing property if and only if the sole bonding map does. Recently in [14], the author discussed connection between limit shadowing property for a continuous map on a compact metric space and that for the shift map on the inverse limit space. In next section we argue relation between ergodic shadowing property (inverse shadowing property) $f$ and $\sigma_{f}$.

\section{Results}

Let $X$ be a compact metric space. Consider $X^{\mathbb{Z}}$ the compact metric space of all two-sided sequences $\left\{x_{n}\right\}_{n \in \mathbb{Z}}$, endowed with the product topology. Let $f: X \rightarrow X$ be a continuous map on $X$. Then the closed subspace $X_{f}=\left\{\left(x_{n}\right): x \in X\right.$, $f\left(x_{n}\right)=x_{n+1}$ for all $\left.n \in \mathbb{Z}\right\}$ of $X^{\mathbb{Z}}$ together with the associated shift map $\sigma_{f}: X_{f} \rightarrow X_{f}$ defined by $\sigma_{f}\left(\left(x_{n}\right)\right)=\left(y_{n}\right)$ with $y_{n}=x_{n+1}$ for all $n \in \mathbb{Z}$ is called the inverse limit space of $f$. Note that, with the metric $\widetilde{d}$ defined by $\widetilde{d}\left(\left(x_{i}\right),\left(y_{i}\right)\right)=$ $\sum_{i \in \mathbb{Z}}\left(d\left(x_{i}, y_{i}\right) / 2^{|i|}\right), X_{f}$ is a compact metric space. For every $i, j \in \mathbb{Z}$ define $i$ the project map $\pi_{i}: X_{f} \rightarrow X$ by $\pi_{i}(\tilde{x})=x_{i}$ for every $\tilde{x} \in X_{f}$ such that $\tilde{x}=\left\{\ldots, x_{i-1}, x_{i}, x_{i+1}, \ldots\right\} . \pi_{i}$ is an open continuous map and satisfies fo $\pi_{i}=\pi_{i} o \sigma_{f}$ for every $i \in \mathbb{Z}$.

Theorem 7. Let $f: X \rightarrow X$ be a continuous surjective map on a compact metric space $X$.

(1) If $f$ has ergodic shadowing property, then the shift map $\sigma_{f}$ on the inverse limit space $X_{f}$ has ergodic shadowing property.

(2) If $f$ has inverse shadowing property, then the shift map $\sigma_{f}$ on the inverse limit space $X_{f}$ has inverse shadowing property.

Proof. Given $\epsilon>0$, suppose that $\operatorname{diam}(X)=\alpha$ and choose $N \in \mathbb{N}$ such that $\left(\alpha / 2^{N-1}\right)<(\epsilon / 8)$. Uniform continuity of $f$ implies existence of some $\gamma>0$ such that if $d(x, y)<\gamma$, then $d\left(f^{i}(x), f^{i}(y)\right)<(\epsilon / 8)$ for $0 \leq i \leq 2 N$.

Since $f$ has ergodic shadowing property, for $\gamma>0$, there exists $\tau>0$ such that every $\tau$-ergodic pseudoorbit is $\gamma$-ergodic shadowed by some points of $X$. Choose $\delta>0$ such that $0<\delta 2^{N}<\tau$. Let $\left\{x^{n}\right\}_{n \in \mathbb{Z}} \subseteq X_{f}$ be $\delta$-ergodic pseudoorbit for $\sigma$. Since $\left\{n \mid d\left(f\left(x_{-N}^{n}\right), x_{-N}^{n+1}\right) \geq \tau\right\} \subseteq$ $\left\{n \mid \widetilde{d}\left(\sigma_{f}\left(x^{n}\right), x^{n+1}\right) \geq \delta\right\}$ the sequence $\left\{x_{-N}^{n}\right\}$ is a $\tau$-ergodic pseudoorbit for $f$. But there exists $y \in X$ such that

$$
\begin{aligned}
\lim _{|m| \rightarrow \infty}( & \operatorname{card}\left(\left\{n \mid d\left(f^{n}(y), x_{-N}^{n}\right) \geq \gamma\right\}\right. \\
& \cap\{-m+1, \ldots, m-1\})) \times m^{-1} \\
= & 0 .
\end{aligned}
$$

Let $y_{i-N}=f^{i}(y)$ for $i \geq 0$ and $y_{i-N} \in f^{-1}\left(y_{i+1-N}\right)$ for $i<0$; then $\tilde{y}=\left(y_{i}\right) \in X_{f}$. It is easy to check that if $n \notin\{n \mid$ $\left.d\left(f^{n}(y), x^{n}\right) \geq \gamma\right\}$, then $n \notin\left\{n \mid \widetilde{d}\left(\sigma^{n}(\tilde{y}), x^{n}\right) \geq \epsilon\right\}$; that is, $\left\{n \mid \widetilde{d}\left(\sigma^{n}(\tilde{y}), x^{n}\right) \geq \epsilon\right\} \subseteq\left\{n \mid d\left(f^{n}(y), x_{-N}^{n}\right) \geq \gamma\right\}$. Therefor (2) shows that $\left\{x^{n}\right\} \subseteq X_{f}$ can be $\epsilon$-ergodic shadowed by $\tilde{y} \in X_{f}$.

Since $f$ has inverse shadowing property, for $\gamma>0$, there exists $\tau>0$ such that, for any $x \in X$ and any $\tau$-method $\varphi$, there is $y \in X$ such that $d\left(f^{k}(x), \varphi_{k}(y)\right)<\gamma$, for all $k \in \mathbb{Z}$. Choose $\delta>0$ such that $0<\delta 2^{N}<\tau$. Suppose that $\tilde{\varphi}: X_{f} \rightarrow$ $\widetilde{\Phi}_{\sigma}(\delta)$ is a $\delta$-method for $\sigma_{f}$; we construct a $\tau$-method for $f$ as follows: let $x \in X$; choose a point $\tilde{x}=\left(x_{i}\right) \in X_{f}$ such that $\pi_{-N}(\tilde{x})=x$. Since $\tilde{\varphi}$ is a $\delta$-method for $\sigma_{f}$, therefor, for $\tilde{x} \in X_{f}, d\left(\sigma_{f}\left(\widetilde{\varphi}_{n}(\tilde{x})\right), \widetilde{\varphi}_{n+1}(\tilde{x})\right)<\delta$. Consider $\varphi: X \rightarrow X^{\mathbb{Z}}$ such that $\varphi(x)=\left\{\varphi_{n}(x)\right\}_{n \in \mathbb{Z}}$, where $\varphi_{n}(x)=\pi_{-N}\left(\tilde{\varphi}_{n}(\tilde{x})\right)$. It is easy to see that $\varphi(x)$ is a $\tau$-pseudoorbit for $f$; thus $\varphi$ is a $\tau$-method for $f$. Since $f$ has inverse shadowing property, for $\pi_{-N}(\tilde{x}) \in X$, such that $\tilde{x} \in X_{f}$, there exists $y \in X$ such that $d\left(f^{n}\left(\pi_{-N}(\tilde{x})\right), \varphi_{n}(y)\right)<\gamma$. Let $\tilde{y} \in X_{f}$ such that $\pi_{-N}(\tilde{y})=$ $y$; then $d\left(\sigma_{f}^{n}(\tilde{x}), \widetilde{\varphi}_{n}(\tilde{y})\right)<\epsilon$; this means that $\sigma_{f}$ has inverse shadowing property.

The converse of the above theorem is not true. We use an example of [13] to show that in general the ergodic shadowing of $\sigma_{f}$ on $X_{f}$ does not imply the ergodic shadowing of $f$ on $X$.

Example 8. Consider $f:[0,1] \rightarrow[0,1]$ with $f(0)=1 / 8$, $f(1 / 4)=1 / 4, f(5 / 16)=1 / 2=f(11 / 16), f(3 / 8)=$ $7 / 16, f(5 / 8)=9 / 16, f(3 / 4)=3 / 4$, and $f(1)=7 / 8$ such that $f$ is linear on each of the subintervals $[0,1 / 4]$, $[1 / 4,5 / 16],[5 / 16,3 / 8],[3 / 8,5 / 8],[5 / 8,11 / 16],[11 / 16,3 / 4]$, and $[3 / 4,1]$. Observe that $f$ is not shadowing as $f \mid[0,1 / 2]$ has no shadowing property; thus $f$ has no ergodic shadowing property. Further, a point $\widetilde{x}=\left(x_{n}\right) \in[0,1]_{f}$ if and only if $x_{n} \in$ $[1 / 4,3 / 4]$ for all $n \in \mathbb{Z}$. Therefor shift map $\sigma_{f}:[0,1]_{f} \rightarrow$ $[0,1]_{f}$ has only two fixed points; thus, by Proposition $6, \sigma_{f}$ has shadowing property and it is known that shift map $\sigma_{f}$ : $[0,1]_{f} \rightarrow[0,1]_{f}$ is topologically mixing (see proposition 7.5 of [9]); therefor $\sigma_{f}$ has ergodic shadowing property. Note that $f$ is not a local homeomorphism on $[0,1]$.

We do not know whether the converse of Theorem 7 in the case of inverse shadowing property holds. But we give an example that $\sigma_{f}: X_{f} \rightarrow X_{f}$ has ISP ${ }^{+}$but $f$ has no $\mathrm{ISP}^{+}$. 
Example 9. Consider $f:[0,1] \rightarrow[0,1]$ with $f(0)=1 / 8$, $f(1 / 4)=1 / 4, f(5 / 16)=1 / 2, f(3 / 8)=7 / 16, f(5 / 8)=$ $9 / 16$, and $f(1)=3 / 8$, such that $f$ is linear on each of the subintervals $[0,1 / 4],[1 / 4,5 / 16],[5 / 16,3 / 8],[3 / 8,5 / 8]$, and $[5 / 8,1]$. Observe that $f$ has no $\mathrm{ISP}^{+}$, but $\sigma_{f}:[0,1]_{f} \rightarrow$ $[0,1]_{f}$ has only one fixed point; thus, by Proposition 4 , it is positive inverse shadowing, $\mathrm{ISP}^{+}$. Note that $f$ is not a local homeomorphism.

In the following theorem we express some conditions under which ergodic shadowing property and inverse shadowing property of $\sigma_{f}$ on $X_{f}$ imply ergodic shadowing property and inverse shadowing property of $f$ on $X$, respectively.

Theorem 10. Let $f$ be a local homeomorphism on a compact metric space $X$.

(1) If the shift map $\sigma_{f}$ on $X_{f}$ has ergodic shadowing property, then $f$ has ergodic shadowing property.

(2) If the shift map $\sigma_{f}$ on $X_{f}$ has inverse shadowing property, then $f$ has inverse shadowing property.

Proof. Given $\epsilon>0$, by ergodic shadowing property of $\sigma_{f}$, there exists $\tau>0$ such that every $\tau$-ergodic pseudoorbit for $\sigma_{f}$ is $\epsilon$-ergodic shadowed by some point in $X_{f}$. Let $\alpha=$ $\operatorname{diam}(X)$; choose $N \in \mathbb{N}$ such that $\left(\alpha / 2^{N-1}\right)<(\tau / 4) . f$ is a local homeomorphism; there exists $\gamma$ with $0<\gamma<(\tau / 4)$ such that $f \mid U: U \rightarrow U$ is a homeomorphism, where $U$ is the $\gamma$-neighborhood of $x$ in $X$. $f$ is uniformly continuous, for $\tau / 8$; there exists $0<\eta<(\tau / 8)$ such that $d(x, y)<\eta$ implies $d\left(f^{j}(x), f^{j}(y)\right)<(\tau / 8)$ for all $j,|j| \leq N$. Suppose that $\left\{x_{n}\right\}_{n \in \mathbb{Z}}$ is an $\eta$-ergodic pseudoorbit for $f$; let $x_{0}^{n}=x_{n}$ and $x^{n}=\left(x_{i}^{n}\right) \in X_{f}$. If $n \notin\left\{n \mid d\left(f\left(x_{n}\right), x_{n+1}\right) \geq \eta\right\}$, then $n \notin\{n \mid$ $\left.\widetilde{d}\left(\sigma_{f}\left(x^{n}\right), x^{n+1}\right) \geq \tau\right\}$. This implies that $\left\{n \mid \widetilde{d}\left(\sigma_{f}\left(x^{n}\right), x^{n+1}\right) \geq\right.$ $\tau\} \subseteq\left\{n \mid d\left(f\left(x_{n}\right), x_{n+1}\right) \geq \eta\right\}$, and therefor $\left\{x^{n}\right\}$ is a $\tau$-ergodic pseudoorbit for $\sigma_{f}$. Since $\sigma_{f}$ has ergodic shadowing property, there exists $\tilde{y}=\left(y_{i}\right)$ in $X_{f}$ with $\lim _{|m| \rightarrow \infty}\left(\left\{n \mid \widetilde{d}\left(\sigma_{f}^{n}(\tilde{y}), x^{n}\right) \geq\right.\right.$ $\epsilon\} \bigcap\{-m+1, \ldots, m-1\} / m)=0$. If $n \notin\left\{n \mid \widetilde{d}\left(\sigma_{f}^{n}(\tilde{y}), x^{n}\right) \geq\right.$ $\epsilon\}$, then $n \notin\left\{n \mid d\left(f^{n}\left(y_{0}\right), x_{0}^{n}\right) \geq \epsilon\right\}$, and we get $\{n \mid$ $\left.d\left(f^{n}\left(y_{0}\right), x_{n}\right) \geq \epsilon\right\} \subseteq\left\{n \mid \widetilde{d}\left(\sigma_{f}^{n}(Y), x^{n}\right) \geq \epsilon\right\}$. Now it is easy using definition to see that $f$ has ergodic shadowing property. Given $\epsilon>0$ by inverse shadowing property of $\sigma_{f}$ there exists $\tau>0$ such that, for every $\tilde{x} \in X_{f}$ and any $\tau$-method $\tilde{\varphi}$ for $\sigma_{f}$, there exists $\tilde{y} \in X_{f}$ such that $\tilde{d}\left(\sigma_{f}^{n}(\tilde{x}), \widetilde{\varphi}_{n}(\tilde{y})\right)<\epsilon$. Let $\alpha=$ $\operatorname{diam}(X)$; choose $N \in \mathbb{N}$ such that $\left(\alpha / 2^{N-1}\right)<(\tau / 4) . f$ is a local homeomorphism; there exists $\gamma$ with $0<\gamma<(\tau / 4)$ such that $f \mid U: U \rightarrow U$ is a homeomorphism, where $U$ is the $\gamma$-neighborhood of $x$ in $X . f$ is uniformly continuous, for $\tau / 8$; there exists $0<\eta<(\tau / 8)$ such that $d(x, y)<\eta$ implies $d\left(f^{j}(x), f^{j}(y)\right)<(\tau / 8)$ for all $j,|j| \leq N$. Let $x \in X$ and let $\varphi: X \rightarrow \Phi_{f}(\delta)$ be a $\gamma$-method for $f$. Define $\tilde{\varphi}: X_{f} \rightarrow X_{f}^{\mathbb{Z}}$ such that $\tilde{\varphi}(\tilde{x})=\left\{\tilde{\varphi}_{n}(\tilde{x})\right\}_{n \in \mathbb{Z}}$ and $\pi_{0}(\tilde{\varphi}(\tilde{x}))=\varphi_{n}(x)$; we have $\widetilde{d}\left(\sigma_{f}\left(\widetilde{\varphi}_{n}(\tilde{x})\right), \widetilde{\varphi}_{n+1}(\tilde{x})\right)<\delta$. Therefor $\widetilde{\varphi}: X_{f} \rightarrow X_{f}^{\mathbb{Z}}$ is a $\delta$ method for $\sigma_{f}$, since $\sigma_{f}$ has inverse shadowing property; thus for $\tilde{x} \in X_{f}$ there exists $\tilde{y} \in X_{f}$ such that $\widetilde{d}\left(\sigma_{f}^{n}(\tilde{x}), \widetilde{\varphi}_{n}(\tilde{y})\right)<\epsilon$; this means that $d\left(f^{n}(x), \varphi_{n}(y)\right)<\epsilon$; that is, $f$ has inverse shadowing property.

\section{Conflict of Interests}

The author declares that there is no conflict of interests regarding the publication of this paper.

\section{References}

[1] S. Y. Pilyugin, Shadowing in Dynamical Systems, vol. 1706 of Lecture Notes in Mathematics, Springer, Berlin, Germany, 1999.

[2] A. Fakhari and F. H. Ghane, "On shadowing: ordinary and ergodic," Journal of Mathematical Analysis and Applications, vol. 364, no. 1, pp. 151-155, 2010.

[3] D. A. Dastjerdi and M. Hosseini, "Sub-shadowings," Nonlinear Analysis: Theory, Methods \& Applications A: Theory and Methods, vol. 72, no. 9-10, pp. 3759-3766, 2010.

[4] R. Li, "On the ergodic shadowing," Communications in Computer and Information Science, vol. 163, pp. 46-53, 2011.

[5] W. Mahavier, "Inverse limits with subsets of $[0,1] \times[0,1]$," Topology and Its Applications, vol. 141, no. 1-3, pp. 225-231, 2004.

[6] W. T. Ingram and W. S. Mahavier, "Inverse limits of upper semi-continuous set valued functions," Houston Journal of Mathematics, vol. 32, no. 1, pp. 119-130, 2006.

[7] L. Chen and S. H. Li, "Shadowing property for inverse limit spaces," Proceedings of the American Mathematical Society, vol. 115, no. 2, pp. 573-580, 1992.

[8] S. H. Li, "Dynamical properties of the shift maps on the inverse limit spaces," Ergodic Theory and Dynamical Systems, vol. 12, no. 1, pp. 95-108, 1992.

[9] M. Denker, C. Grillenberger, and K. Sigmund, Ergodic Theory on Compact Spaces, Lecture Notes in Mathematics, Springer, New York, NY, USA, 1976.

[10] L. Block and E. M. Coven, "Topological conjugacy and transitivity for a class of piecewise," Transactions of the American Mathematical Society, vol. 300, no. 1, pp. 297-306, 1987.

[11] P. Walters, An Introduction to Ergodic Theory, vol. 79 of Graduate Texts in Mathematics, Springer, New York, NY, USA, 1982.

[12] B. Honary and A. Zamani Bahabadi, "Asymptotic average shadowing property on compact metric spaces," Nonlinear Analysis: Theory, Methods \& Applications, vol. 69, no. 9, pp. 2857-2863, 2008.

[13] E. Shah and T. K. Das, "On shadowing property for inverse limit spaces," Mathematica Slovaca, vol. 5, pp. 95-100, 2008.

[14] R. Gu and Y. Sheng, "APOTP for the inverse limit spaces," Applied Mathematics A: Journal of Chinese Universities Series B, vol. 21, no. 4, pp. 473-478, 2006. 


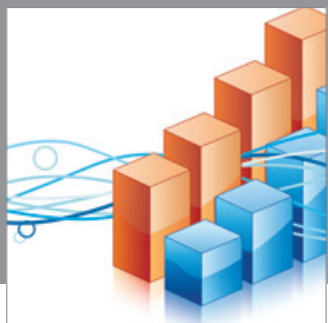

Advances in

Operations Research

mansans

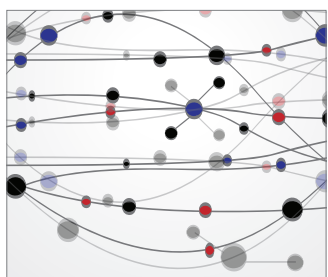

The Scientific World Journal
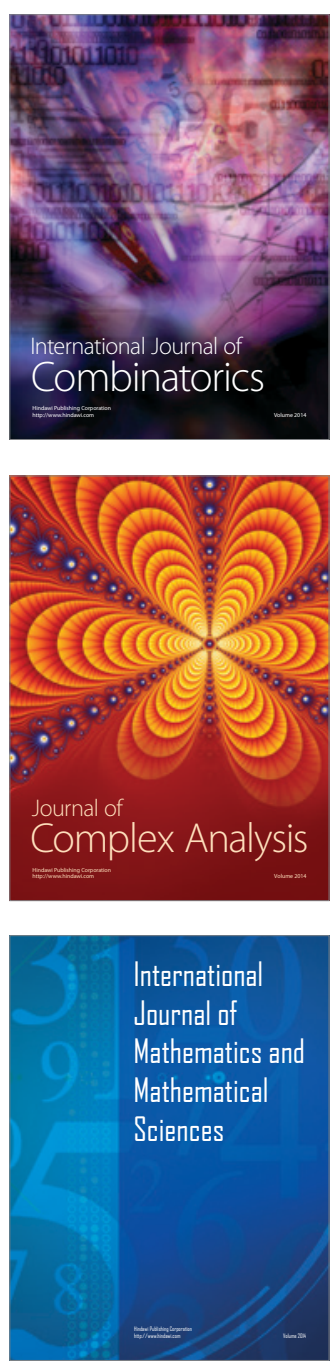
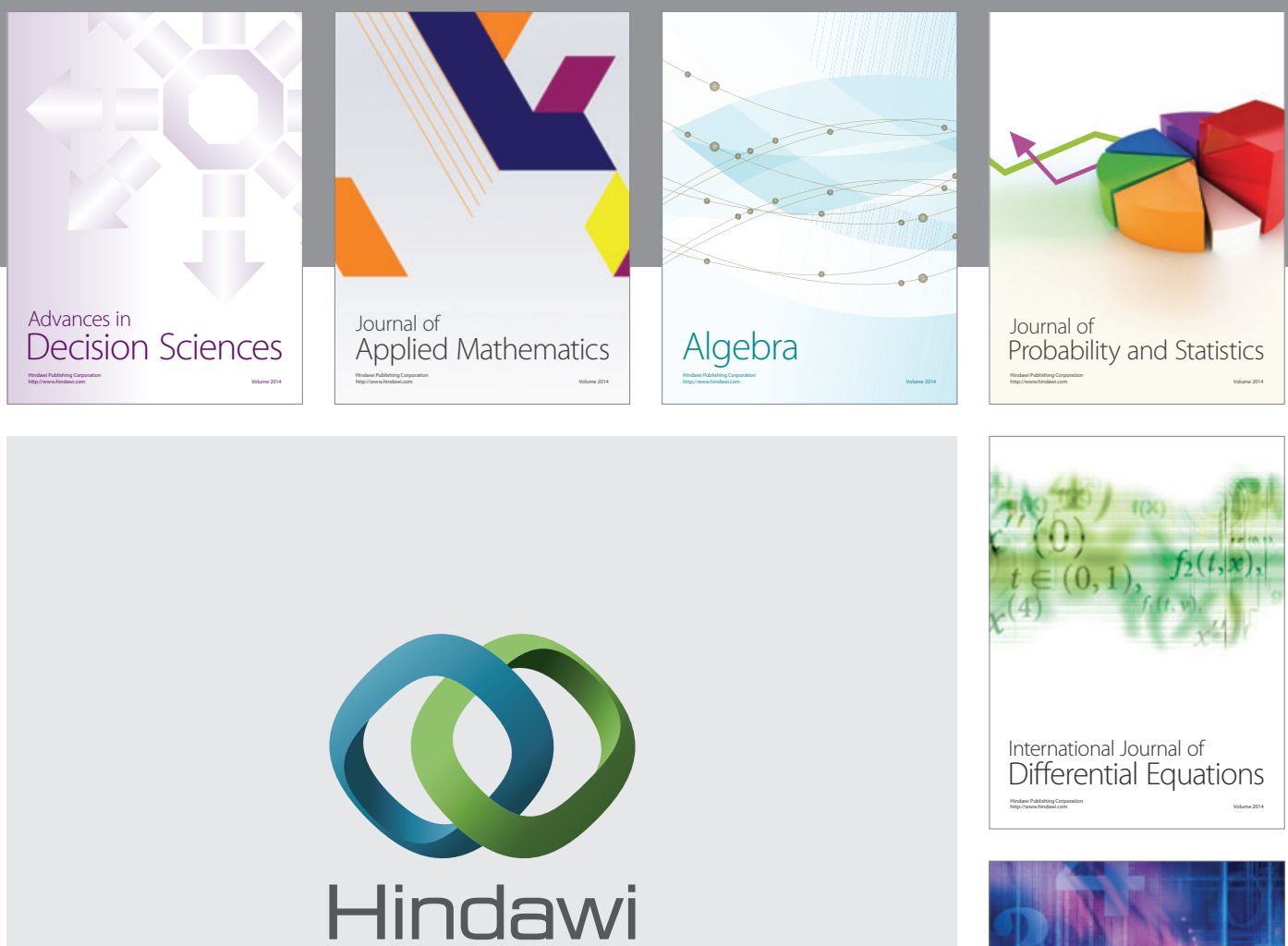

Submit your manuscripts at http://www.hindawi.com
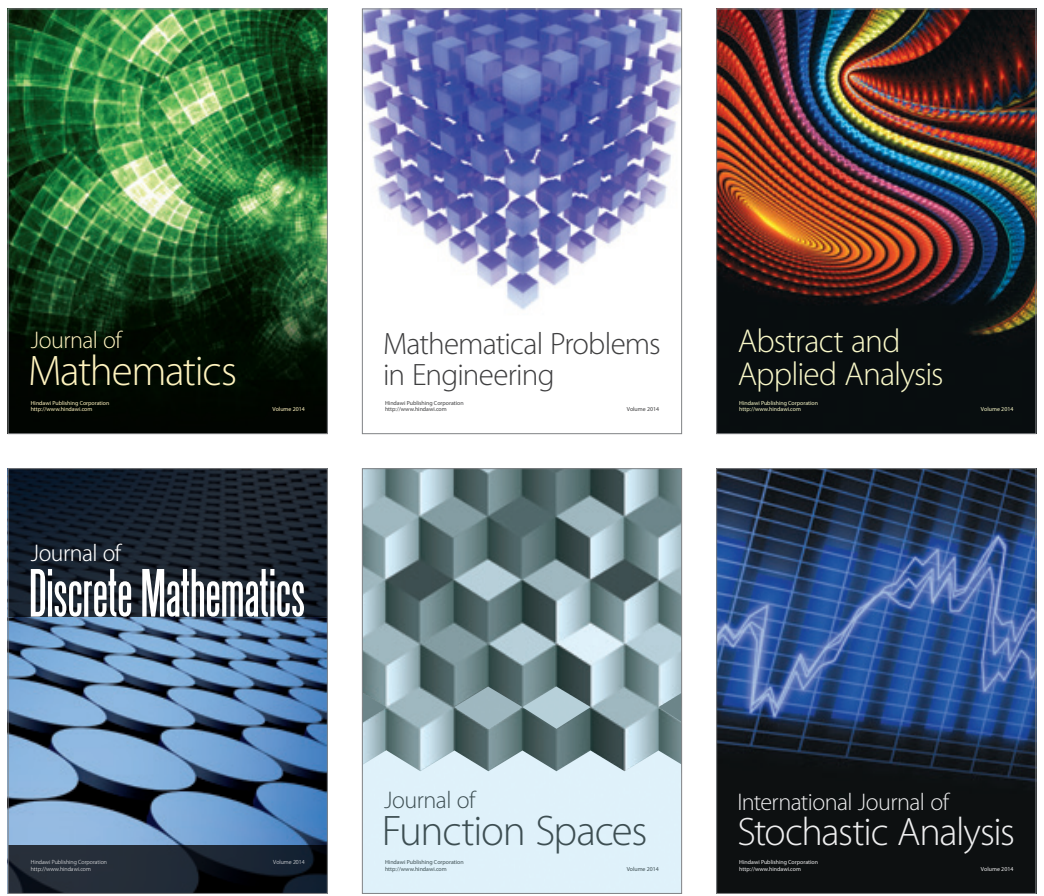

Journal of

Function Spaces

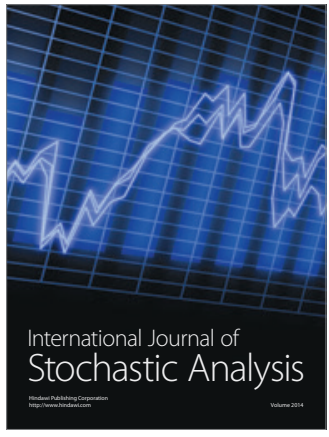

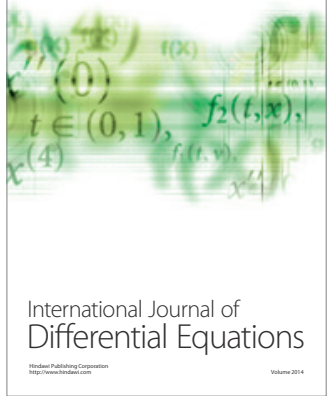
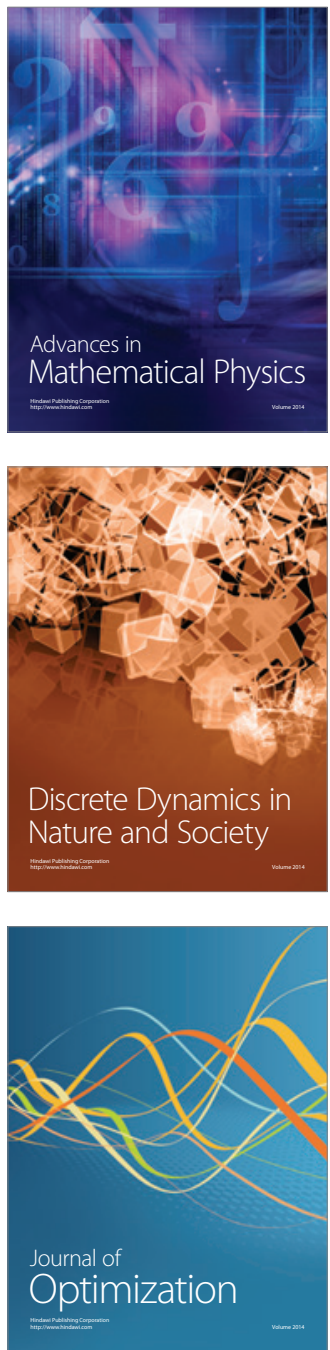